\title{
TITLE:
}

\section{A note on disjoint arborescences}

\author{
AUTHOR(S):
}

\section{Fujishige, Satoru}

\section{CITATION:}

Fujishige, Satoru. A note on disjoint arborescences. Combinatorica 2010, 30(2): 247-252

\section{ISSUE DATE:}

2010-03

URL:

http://hdl.handle.net/2433/130690

\section{RIGHT:}

The original publication is available at www.springerlink.com; This is not the published version. Please cite only the published version.; この 論文は出版社版でありません。引用の際には出版社版をご確認ご利用 ください。 
Title: A Note on Disjoint Arborescences

Author: SATORU FUJISHIGE

Affiliation: Research Institute for Mathematical Sciences, Kyoto University,

Kyoto 606-8502, Japan

E-mail: fujishig@kurims.kyoto-u.ac.jp

Abbreviated Title: A Note on Disjoint Arborescences

Mathematics Subject Classification: Primary: 05C70; Secondary: 05C20, 05C40, 90C27 


\title{
A Note on Disjoint Arborescences
}

\author{
SATORU FUJISHIGE \\ Research Institute for Mathematical Sciences \\ Kyoto University, Kyoto 606-8502, Japan \\ fujishig@kurims.kyoto-u.ac.jp
}

July 4, 2008; revised October 10, 2008

\begin{abstract}
Recently Kamiyama, Katoh, and Takizawa have shown a theorem on packing arc-disjoint arborescences that is a proper extension of Edmonds' theorem on disjoint spanning branchings. We show a further extension of their theorem, which makes clear an essential rôle of a reachability condition played in the theorem. The right concept required for the further extension is "convexity" instead of "reachability."
\end{abstract}

\section{Introduction: a theorem of Kamiyama, Katoh, and Takizawa}

Recently Kamiyama, Katoh, and Takizawa [3] have shown a theorem (KKT theorem for short in the sequel) on packing arc-disjoint arborescences that is a proper extension of Edmonds' theorem [2] on disjoint spanning branchings, which is described as follows. (The precise definitions of terms used here will be given later.)

Let $G=(V, A)$ be a directed graph with a vertex set $V$ and an arc set $A$. For any vertex $v \in V$ we denote by $R_{G}^{+}(v)$ the set of vertices reachable from $v$ by directed paths in $G$. Given roots $r_{i}(i \in I)$, KKT theorem gives a characterization of the existence of a set of arc-disjoint arborescences $H_{i}(i \in I)$ such that for each $i \in I$ arborescence $H_{i}$ has a root $r_{i}$ and exactly spans $R_{G}^{+}\left(r_{i}\right)$.

In this note we show a further extension of KKT theorem, which makes clear an essential rôle played by a reachability condition in the theorem. The right concept required for the further extension is "convexity" instead of "reachability."

For more information about disjoint arborescences, their extensions, and related topics see $[4$, Part V] and [1]. 


\section{An extension of KKT theorem}

Let $G=(V, A)$ be a directed graph with a vertex set $V$ and an arc set $A$. Each arc $a \in A$ has a tail denoted by $\partial^{+} a$ and a head denoted by $\partial^{-} a$. For any vertex $v$ the in-degree of $v$ is equal to the number of arcs that have $v$ as their heads. A branching in $G$ is a subgraph $H=(U, B)$ of $G$ without any cycle such that every vertex $u$ in $U$ has in-degrees at most one in $H$. Each connected component of branching $H$ has a unique vertex, called a root, that has the in-degree equal to zero in $H$. A connected branching is called an arborescence, which has a single root.

For any vertex $v \in V$ we denote by $R_{G}^{+}(v)$ the set of vertices reachable from $v$ by directed paths in $G$ and by $R_{G}^{-}(v)$ the set of vertices from which $v$ is reachable by a directed path in $G$. Also define for any $W \subseteq V$

$$
R_{G}^{+}(W)=\bigcup\left\{R_{G}^{+}(v) \mid v \in W\right\}, \quad R_{G}^{-}(W)=\bigcup\left\{R_{G}^{-}(v) \mid v \in W\right\} .
$$

A vertex subset $W$ is called a convex set in $G$ if we have $W=R_{G}^{+}(W) \cap R_{G}^{-}(W)$, i.e., for every directed (possibly closed) path $P$ from a vertex in $W$ to a vertex in $W$ all the intermediate vertices of $P$ also lie in $W$. The concept of convexity plays an essential rôle in our result, which replaces the rôle of reachability from roots in KKT theorem [3]. It should be noted that for any convex set $U$ in $G$ and the vertex set $W$ of any strongly connected component of $G$ that satisfy $U \cap W \neq \emptyset$, we must have $U \supseteq W$.

Suppose that we are given a finite index set $I$ and, for each $i \in I$, a specified vertex $r_{i} \in V$. Here we may allow $r_{i}=r_{j}$ for some distinct $i, j \in I$. For each $i \in I$ we are also given a convex set $U_{i} \subseteq V$ such that $r_{i} \in U_{i}$. For any $v \in V$ define

$$
I(v)=\left\{i \in I \mid v \in U_{i}\right\} .
$$

We assume that $I(v) \neq \emptyset$ for all $v \in V$.

Now we are ready to state our main theorem, which is an extension of KKT theorem. It should be noted that replacing $U_{i}$ by $R_{G}^{+}\left(r_{i}\right)$ for all $i \in I$ in our theorem yields KKT theorem. Our proof employs KKT theorem recursively. For any vertex subset $Z \subseteq V$ denote by $G[Z]$ the subgraph of $G$ induced by $Z$.

Theorem 2.1: The following two statements are equivalent.

(a) There exist arc-disjoint arborescences $H_{i}=\left(U_{i}, B_{i}\right)(i \in I)$ such that for each $i \in I$ arborescence $H_{i}$ has a root $r_{i}$.

(b) For each $v \in V$ there exist arc-disjoint directed paths $P_{i}(i \in I(v))$ such that for each $i \in I(v)$ path $P_{i}$ is from $r_{i}$ to $v$.

(Proof) $((a) \Rightarrow(b))$ : This implication is easy.

((b) $\Rightarrow($ a)): Suppose (b) holds.

Consider the decomposition of graph $G$ into strongly connected components, which defines a partial order $\preceq$ on the set of strongly connected components as follows. For two 
strongly connected components $H$ and $H^{\prime}$ we have $H \preceq H^{\prime}$ if and only if there exists a directed path from $H^{\prime}$ to $H$. Let $W \subseteq V$ be the vertex set of a strongly connected component that is minimal with respect to the partial order $\preceq$. In other words, $W$ is the vertex set of a strongly connected component in $G$ such that $R_{G}^{+}(W)=W$.

Define

$$
\begin{aligned}
& I(W)=\bigcup\{I(v) \mid v \in W\}\left(=\left\{i \in I \mid W \subseteq U_{i}\right\}\right), \\
& U_{i}(W)=U_{i} \cap R_{G}^{-}(W) \quad(i \in I(W)), \\
& V(W)=\bigcup\left\{U_{i}(W) \mid i \in I(W)\right\} .
\end{aligned}
$$

Then consider the subgraph $\hat{G}=G[V(W)]$ of $G$ induced by $V(W)$. Because of the convexity of $U_{i}(i \in I)$, definitions (2.3)-(2.5), and assumption (b) we can show the following two facts.

Fact 1: For each $i \in I(W) U_{i}(W)$ is exactly the set of vertices that can be reached from $r_{i}$ by directed paths in $\hat{G}$, i.e., $R_{\hat{G}}^{+}\left(r_{i}\right)=U_{i}(W)$.

Fact 2: For any $v \in V(W)$ and any directed path $P$ in $G$ from $r_{i}(i \in I(W))$ to $v$ all the intermediate vertices of $P$ lie in $U_{i}(W)$.

It follows from these two facts that assumption (b) (appropriately modified) also holds for graph $\hat{G}$ with index set $I(W)$ and convex (reachable) sets $R_{\hat{G}}^{+}\left(r_{i}\right)=U_{i}(W)(i \in I(W))$. More precisely, the following (*) holds.

(*) for each $v \in V(W)$ there exist arc-disjoint directed paths $P_{i}(i \in I(v) \cap I(W))$ such that for each $i \in I(v) \cap I(W)$ path $P_{i}$ is from $r_{i}$ to $v$ in $\hat{G}$.

Hence from KKT theorem there exist arc-disjoint arborescences $\hat{H}_{i}=\left(U_{i}(W), \hat{B}_{i}\right)(i \in$ $I(W))$ such that each arborescence $\hat{H}_{i}(i \in I(W))$ has a root $r_{i}$.

Define

$$
B_{i}^{W}=\hat{B}_{i} \cap \delta^{-} W \quad(i \in I(W)),
$$

where $\delta^{-} W$ is the set of arcs $a \in A$ with $\partial^{-} a \in W$. (Here note that we may have $\partial^{+} a \in W$.) For all $i \in I \backslash I(W)$ define $B_{i}^{W}=\emptyset$. Then put

$$
\begin{aligned}
& G \leftarrow G \backslash W, \\
& U_{i} \leftarrow U_{i} \backslash W \quad(i \in I), \\
& I \leftarrow I \backslash\left\{i \in I \mid r_{i} \in W\right\},
\end{aligned}
$$

where $G \backslash W$ is the graph obtained by removing from $G$ the vertices of $W$ and the arcs incident to $W$. Note that if $G \backslash W$ has desired arc-disjoint arborescences $H_{i}^{\prime}=\left(U_{i} \backslash W, B_{i}^{\prime}\right)$ $(i \in I)$ restricted on $G \backslash W$, then $H_{i}=\left(U_{i}, B_{i}^{\prime} \cup B_{i}^{W}\right)(i \in I)$ are desired ones for $G$. It should also be noted that $U_{i} \backslash W(i \in I)$ are convex sets in the original graph $G$ and hence in the new $G$ as well. Since $U_{i} \backslash W(i \in I)$ are convex sets in the original graph $G$, 
directed paths within $U_{i} \backslash W$ in the original $G$ are also directed path in the new $G$. Hence assumption (b) also holds for the new $G, I, U_{i}(i \in I)$, and $r_{i}(i \in I)$.

Repeat this process until $G$ becomes empty. Let $W_{1}, \cdots, W_{k}$ be the sequence of $W \mathrm{~s}$ chosen in the repeated above-mentioned process.

Define for each $i \in I$

$$
B_{i}=\bigcup\left\{B_{i}^{W_{\ell}} \mid \ell=1, \cdots, k\right\},
$$

where $B_{i}^{W_{\ell}}$ is defined to be $B_{i}^{W}$ for $W=W_{\ell}$. We can easily see that $H_{i} \equiv\left(U_{i}, B_{i}\right)$ $(i \in I)$ are desired arborescences with roots $r_{i}(i \in I)$, one for each corresponding $H_{i}$.

Note that the proof given above leads us to a polynomial algorithm for finding arcdisjoint arborescences that span specified convex sets with roots by using the algorithm in [3].

We can also show the following. Define $I^{\prime}(v)=\left\{i \in I(v) \mid r_{i} \neq v\right\}$ for all $v \in V$.

Theorem 2.2: The following two statements are equivalent to (a) (and (b)) in Theorem 2.1.

(c) For any vertex subset $Z \subset V$

$$
\left|\Delta^{-} Z\right| \geq\left|\left\{i \in I(Z) \mid r_{i} \notin Z\right\}\right|,
$$

where $\Delta^{-} Z$ denotes the set of arcs $a \in A$ such that $\partial^{+} a \notin Z$ and $\partial^{-} a \in Z$.

(d) There exist spanning trees $T_{i}=\left(U_{i}, E_{i}\right)$ of $G\left[U_{i}\right](i \in I)$ such that $E_{i}(i \in I)$ are pairwise disjoint and every vertex $v \in V$ has in-degree equal to $\left|I^{\prime}(v)\right|$ in the union of $T_{i}(i \in I)$ (as a subgraph $H=\left(V, \cup_{i \in I} E_{i}\right)$ of $\left.G\right)$.

(Proof) We show the implications (c) $\Rightarrow$ (b) ((a)) $\Rightarrow$ (d) $\Rightarrow$ (c).

$((\mathrm{c}) \Rightarrow(\mathrm{b}))$ : Let $v$ be any vertex in $V$. Consider any $Z \subset V$ with $v \in Z$ in (c). Then it follows from (c) (with any such $Z$ ) and the max-flow min-cut theorem that (b) for $v$ holds.

$((b) \Rightarrow(d))$ : This is easy since (a) and (b) are equivalent.

$((\mathrm{d}) \Rightarrow(\mathrm{c}))$ : Let $Z$ be any subset of $V$. Denote by $A_{H}[Z]$ the set of $\operatorname{arcs} a$ in $H$ with $\partial^{+} a, \partial^{-} a \in Z$. Then we have

$$
\left|\Delta^{-} Z\right| \geq \sum_{v \in Z}\left|I^{\prime}(v)\right|-\left|A_{H}[Z]\right| \geq\left|\left\{i \in I(Z) \mid r_{i} \notin Z\right\}\right|,
$$

where the second inequality follows from the fact that $\left|E_{i} \cap A_{H}[Z]\right| \leq\left|U_{i} \cap Z\right|-1$ for all $i \in I(Z)$. (Note that $\left|A_{H}[Z]\right|=\sum_{i \in I(Z)}\left|E_{i} \cap A_{H}(Z)\right| \leq \sum_{i \in I(Z)}\left|U_{i} \cap Z\right|-|I(Z)|=$ $\sum_{v \in Z}|I(v)|-|I(Z)|$.) Hence (2.11) holds.

It should be noted that because of (d) in Theorem 2.2 a problem of finding minimumweight arc-disjoint arborescences that span given convex sets with roots can be solved in polynomial time. 


\section{Acknowledgments}

I am grateful to Naoyuki Kamiyama for pointing out an error in statement (d) of Theorem 2.2 in an earlier version of this note. Thanks are also due to András Frank for useful discussions and comments on disjoint arborescences and to the referee for his careful reading. The present research was supported by a Grant-in-Aid from the Ministry of Education, Culture, Sports, Science and Technology of Japan.

\section{References}

[1] K. Bérczi and A. Frank: Variations for Lovász' submodular ideas. Technical Report TR-2008-7, The Egerváry Research Group on Combinatorial Optimization (June 2008).

[2] J. Edmonds: Edge-disjoint branchings. In: Combinatorial Algorithms (B. Rustin, ed.) (Academic Press, 1973), pp. 91-96.

[3] N. Kamiyama, N. Katoh, and A. Takizawa: Arc-disjoint in-trees in directed graphs. Proceedings of the 19th Annual ACM-SIAM Symposium on Discrete Algorithms (2008), pp. 518-526; also to appear in Combinatorica.

[4] A. Schrijver: Combinatorial Optimization-Polyhedra and Efficiency (Springer, 2003). 